\title{
Dynamic brain PET/MR using TOF reconstruction
}

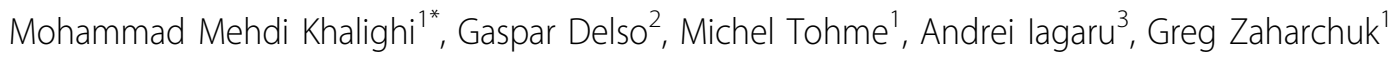

From PSMR 2015: 4th Conference on PET/MR and SPECT/MR

La Biodola, Isola d'Elba, Italy. 17-21 May 2015

${ }^{1}$ GE Healthcare, USA
In a functional PET/MR study, it is difficult to get good temporal resolution of activity distribution from PET images because of the need to image for a certain length of time to get sufficient count statistics (image SNR). Time-of-flight (TOF) reconstruction can be used to increase PET images SNR and therefore increase the temporal resolution. Five patients were injected with $410 \pm 80 \mathrm{MBq}$ of FDG and scanned $140 \pm 30 \mathrm{~min}-$ utes post-injection on a simultaneous TOF-enabled PET/MR scanner. PET images were reconstructed with and without TOF. TOF reconstruction shows faster convergence while it achieves a temporal SNR improvement of 5-45\% (25 $\pm 5 \%$ ) compared to non-TOF reconstruction. With this additional SNR gain, frame durations as short as 30s are possible while preserving reasonable image quality. This in turn effectively increases the temporal resolution of dynamic brain studies using simultaneous PET/ MR imaging.

Authors' details

${ }^{1}$ GE Healthcare, USA. ${ }^{2}$ UniversitätsSpital Zürich, Switzerland. ${ }^{3}$ Stanford University, USA.

Published: 18 May 2015

doi:10.1186/2197-7364-2-S1-A60

Cite this article as: Khalighi et al:: Dynamic brain PET/MR using TOF reconstruction. EJNMMI Physics 2015 2(Suppl 1):A60.

\section{SpringerOpen ${ }^{\circ}$}

(C) 2015 Khalighi et al; licensee Springer. This is an Open Access article distributed under the terms of the Creative Commons Attribution License (http://creativecommons.org/licenses/by/4.0), which permits unrestricted use, distribution, and reproduction in any medium, provided the original work is properly cited. 